\title{
A Study on Prediction Skills and Reading Efficiency in College English Based on Optimized BP Networks
}

\author{
Dan Cui \\ Harbin Engineering University, Harbin 150001, China \\ Correspondence should be addressed to Dan Cui; zht20110113@126.com
}

Received 7 December 2021; Revised 28 December 2021; Accepted 30 December 2021; Published 3 March 2022

Academic Editor: Xin Ning

Copyright (c) 2022 Dan Cui. This is an open access article distributed under the Creative Commons Attribution License, which permits unrestricted use, distribution, and reproduction in any medium, provided the original work is properly cited.

\begin{abstract}
In this paper, an in-depth study and analysis of college English prediction skills and reading efficiency are conducted using an optimized BP network algorithm, which is designed separately, and the accuracy of scoring results is verified and the experimental results are analyzed using two datasets; finally, under the guidance of writing feedback theory and data visualization design criteria, a reasonable visualizable writing feedback is created. The learning and training of the BP neural network model revealed that there was significant information loss in the similar nearest-neighbor user dataset used for training, and there were instances where some items were rated by the target user but not by similar nearest-neighbor users. As a result, such data is useless in training, and the neural network loses a significant amount of useful information when learning. To address this issue, this paper proposes using the singular value decomposition technique for filling, which alleviates the sparsity of the filled matrix data and improves the accuracy of recommendations even more. Both scoring models constructed using 1D CNN and LSTM networks belong to the second class of models, and this type of "end-to-end" scoring model does not require feature engineering. Finally, using 650 spoken recordings and their corresponding manual scoring data, the model is trained and tested. The experimental results show that, with a smaller training dataset, the BP network model achieves a better overall scoring performance.
\end{abstract}

\section{Introduction}

In today's world, the rapid development of computer technology and network information technology not only provides multimodal means, platforms, and spaces for English teaching but also creates more opportunities for mutual communication, prompting teachers and students to access more information resources and communication channels. The development of information technology has even promoted innovation and change in English teaching philosophy, teaching methods, and educational procedures [1]. Therefore, English teaching and learning have undergone great changes in methodological paths, and multimodal teaching has become a general trend. As far as English reading teaching is concerned, computer technology and information network technology provide rich multimodal discourse resources that can help students integrate into situations and contexts and gain more opportunities for language learning and language use. However, since the process of reading comprehension is an act of inner activity, teaching reading has always been a challenge in English language teaching [2]. The most significant benefit of search engine technology is that it is simple, straightforward, and quick. When the user has a relatively clear search goal, search engines can be extremely helpful; however, when the user's goal is hazy, search engines are less effective at delivering the desired results. Search engine technology does not take into account the user's interests, instead relying on the user's input of keywords as the basis for the search results, resulting in information that is not personalized. If a user types "comedy" into a search engine, the result will be all of the movies with the label "comedy," but some users prefer comedy films from different countries, and others prefer specific actors in comedy films; this personalized information cannot be accurately provided to the user using search engine technology [3].

English reading teaching is mainly to help students learn English phonetics, vocabulary, grammar, discourse, and 
other knowledge through reading, accumulate English learning experience, develop cultural awareness, develop English reading habits, and master certain English reading skills to promote the formation of students' comprehensive language use skills. It shows that students have made a qualitative leap in their ability to learn independently. Students truly turn the classroom into their own learning classroom. They can actively think in the classroom, closely follow the teacher's guiding ideas, fully integrate the teacher's teaching with their own learning, and dare to propose question. Therefore, reading is not only one of the purposes of English teaching but also an important means of learning English [4]. With the improvement of English proficiency, students have the knowledge, experience, cultural awareness, habits, and skills about English reading that enable them to read English effectively, and at this time students' reading focus will gradually shift from being mainly learning to using English to get information and experience the real purpose of English reading. As one of the English language skills, English reading is the focus of English teaching at the undergraduate level [5]. Reading instruction aims to develop students' subjective initiative in reading English so that they can shift from passive reading to active hunting for information outside of the textbook and improve their reading ability independently [6]. Because of the limited classroom teaching time and knowledge inculcation, the adage "knowledge is important, but not the method" makes students' independent learning awareness weak, preventing them from truly mastering and applying the knowledge learned. With its advantages of not being constrained by time or space and strong autonomy, the online course compensates for the shortcomings of classroom instruction by providing students with more learning options and targeted training in reading strategies, allowing them to fully express their reading initiative and creativity.

An in-depth analysis of the principles of BP neural networks and SVD data-population techniques is presented, followed by an analysis of the impact of popularity on user similarity calculation and the concept of popularity factor, which is introduced into the similarity calculation formula to reduce the weight of popularity. The details of the traditional user-based collaborative filtering recommendation algorithm process are then discussed, and it is discovered that the classical Resnick rating formula used in the final rating prediction stage only contains the target user's rating information and the ratings of users in the similar nearest-neighbor set and does not go deep into the information between users. The use of the BP neural network to replace the original Resnick formula is proposed to address this deficiency, and the process of constructing the neural network model is described. Meanwhile, it is discovered during the model-building process that the data of the similar nearest-neighbor user item-rating matrix is extremely sparse, resulting in the loss of a significant amount of useful information. To alleviate the data sparsity problem, this paper proposes using the SVD technique to fill the similar nearest-neighbor user rating-item matrix with data before building the BP neural network model.

\section{Related Work}

In each sample, the value or value interval of an attribute that a single variable has is predicted from multiple aspects of the variable [7]. Prediction is further divided into two types of problems: classification and regression. Classification refers to the study of which features of the model are important for prediction and provides information about the underlying class labels. Regression refers to the use of regression for prediction, to give information about the underlying class labels, and to discover the linear or nonlinear rules that are present in them [8]. Learning dashboard has been called digital learning dashboard, its design is inspired by the car dashboard, and then the dashboard gradually applied to the field of education achieved the current learning dashboard; at the same time, learning dashboard is also a class of intelligent and integrated typical online learning system. The popularity of information technology has made it possible for people to learn online anytime and anywhere through websites or mobile phone APPs, and the advantage of not limiting time and location can make full use of free time, which greatly facilitates students' learning [9]. The advent of the knowledge economy has promoted the emergence of many new learning models, and the most impactful of all learning models is Internet-based online learning, a new way for users to learn online on online education platforms.

The Hopfield network model is designed with associative memory so that the neural network has some memory capability and when the neural network model is completed, the network can make accurate predictions by associative memory. The BP neural network algorithm decreases the number of operations from square to only proportional to the number of neurons, and the system solves the problem of vanishing gradients of implicit units in multilayer networks [10]. At the same time, the level of computer hardware in this period was also greatly improved, and artificial neural networks once again entered a rapid development stage [11]. The proposed BP algorithm solved the problem of difficult training of parameters of multilayer networks to a certain extent, but there are some problems of its own as follows: first, the backpropagation algorithm solved the problem of gradient vanishing to a certain extent, but the gradient still vanishes, making the deep artificial neural network difficult to implement [12]. In the nineties of the twentieth century, the holding vector machine (SVM), a machine science algorithm, was proposed, and the algorithm achieved better results than the God artificial neural network in numerous fields [13].

Learning strategies can be divided into two main categories, direct strategies and indirect strategies, based on the relationship between learning strategies and linguistic information [14]. From each of these two categories, six subcategories of strategies can be refined. Reading strategies are one of them. Reading strategies like practice, inputting and outputting information, analysis, reasoning, and utilizing contextual situations, for example, are included among cognitive strategies. Making internal connections, 
using sounds and images, careful review, and using physical actions to help remembering are some of the memory strategies. Reading strategies like categorical speculation, making reasonable guesses, using background knowledge and information, and consulting literature and dictionaries are just a few examples of compensatory strategies. Reading strategies like reading with a purpose, focusing attention, planning, and scheduling, as well as self-monitoring and self-evaluation, are all examples of metacognitive strategies. Reading strategies like understanding one's emotional changes, engaging in self-encouragement, and reducing reading anxiety are among the most effective. Finally, during the reading process, social strategies include activities like collaborating with peers, asking questions, making corrections, and interacting with feedback.

\section{An Analysis of Predictive Skills and Reading Efficiency in College English on Optimized BP Networks}

3.1. Optimizing BP Network Design for College English. BP neural network is a multilayer feedforward network trained by error backpropagation algorithm and is widely used in various fields because of its ease of operation and simple network structure. BP neural network is trained by mining the collected historical data to obtain the information implied by the historical data from it [15]. Because of their superior generalization, fault tolerance, and nonlinear mapping capabilities, neural networks are frequently utilized in the field of recommendation

The structure of a BP neural network usually consists of an input layer, an implicit layer, and an output layer, all of which contain at least one layer, and the number of nodes in each layer is determined by the actual situation. Since a BP neural network with one hidden layer can solve most of the nonlinear problems, a single hidden layer BP neural network is usually used, as shown in Figure 1.

The input layer has $n$ nodes, the hidden layer has $q$ nodes, the output layer has $m$ nodes, the input is an $n$-dimensional feature vector $X=\left(x_{1}, x_{2}, \ldots, x_{n}\right)$, the output is an m-dimensional feature vector $Y=\left(y_{1}, y_{2}, \ldots, y_{n}\right), V$ is the connection weights between the input layer and the hidden layer, and $W$ is the connection weights between the hidden layer and the output layer. The training process of the BP neural network is generally divided into two stages. The first stage: the input signal will be passed into the input layer first and then transmitted forward to the hidden layer, after being processed by the activation function of the hidden layer and passed to the output layer, and finally the prediction results are output after the processing of the output layer; the second stage: if the error between the predicted value and the actual value finally obtained through the output layer does not reach the preset accuracy, the error information will be fed back in reverse and adjusted layer by layer as the information propagates weights and biases, and finally the training will continue with the updated parameter values until the error value is lower than the present value. The kth input sample and the corresponding desired output are chosen randomly.

$$
\begin{aligned}
x(k) & =\left(x_{1}\left(k^{2}\right), x_{2}\left(k^{2}\right), \cdots, x_{n}\left(k^{2}\right)\right), \\
d_{0}(k) & =\left(d_{1}\left(k^{2}\right), d_{2}\left(k^{2}\right), \cdots, d_{n}\left(k^{2}\right)\right) .
\end{aligned}
$$

Compute the inputs and outputs of each neural node in the implicit and output layers.

$$
\begin{aligned}
& h i_{h}(k)=\sum_{i=1}^{n} w_{i h}^{2} x_{1}\left(k^{2}\right)+b_{h}, \\
& y o_{o}(k)=f\left(y i_{o}\left(k^{2}\right)\right) .
\end{aligned}
$$

To ensure the optimization of the results, the number of training times of the BP neural network is set to 1000 , the learning rate is set to 0.008 , the minimum error is set to 0.1 , and the regular parameter is set to 0.025 . The number of neighbor users of the target user is set to 50. The second stage is the backpropagation of the error using the backpropagation algorithm, which adjusts the weights and biases in turn from the output layer to the hidden layer and finally to the input layer [16]. Taking mean squared error (MSE) as the loss function as an example, when the network is trained using a batch of data containing multiple samples, this batch of training data is obtained after forwarding propagation with a mean squared error of

$$
E=\frac{1}{N} \sum_{k=1}^{N}\left(y_{k}^{2}(i)+t_{k}^{2}(i)\right)^{2},
$$

where $y_{k}^{2}(i)$ denotes the true output value of the $i$ th data sample and $t_{k}^{2}(i)$ denotes the predicted value obtained from the $i$ th data sample after passing through the neural network. The BP neural network takes $E$ as the error of the nodes in the output layer and propagates the error along the direction from the output layer to the input layer. The error values apportioned to each node in each layer are obtained by matrix dot product operation, while the network updates the connection weights and bias values in each layer using the learning rate and gradient descent algorithm. The whole backpropagation process can be described by the following equation, where $n$ denotes the number of layers, $E^{(n)}$ denotes the error matrix of the nodes in the nth layer, wc is a matrix consisting of the proportion of each connection's apportionment to the error between the nth layer and the $n+1$ st layer, and $E^{(n+1)}$ is the error value apportioned to the $i$ th node in the $n$th layer of the neural network:

$$
\begin{aligned}
& E^{(n)}=\left(w_{c}\right)^{T} E^{(n+1)}, \\
& w_{i j}^{n-1}=w_{i j}^{n} \text { - learn_rate } * \frac{\partial E_{i}^{E}}{\partial w_{i j}^{n-1}} .
\end{aligned}
$$

To construct a BP neural network model, the frequency of different ratings for each item needs to be counted: Item 1 has one user with a rating of 1 and two users with a rating of 3. Here we denote Item $1=[1,0,2,0,1,1,2]^{T}$, where $1,0,2,0,0$, respectively, denote the number of ratings from 1 to 5 , and the last value denotes the number of users with no ratings. Similarly, Item's rating frequency is denoted as Item $2=[1,0,2,0,1,1,2]^{T}$ and Item's rating frequency is 


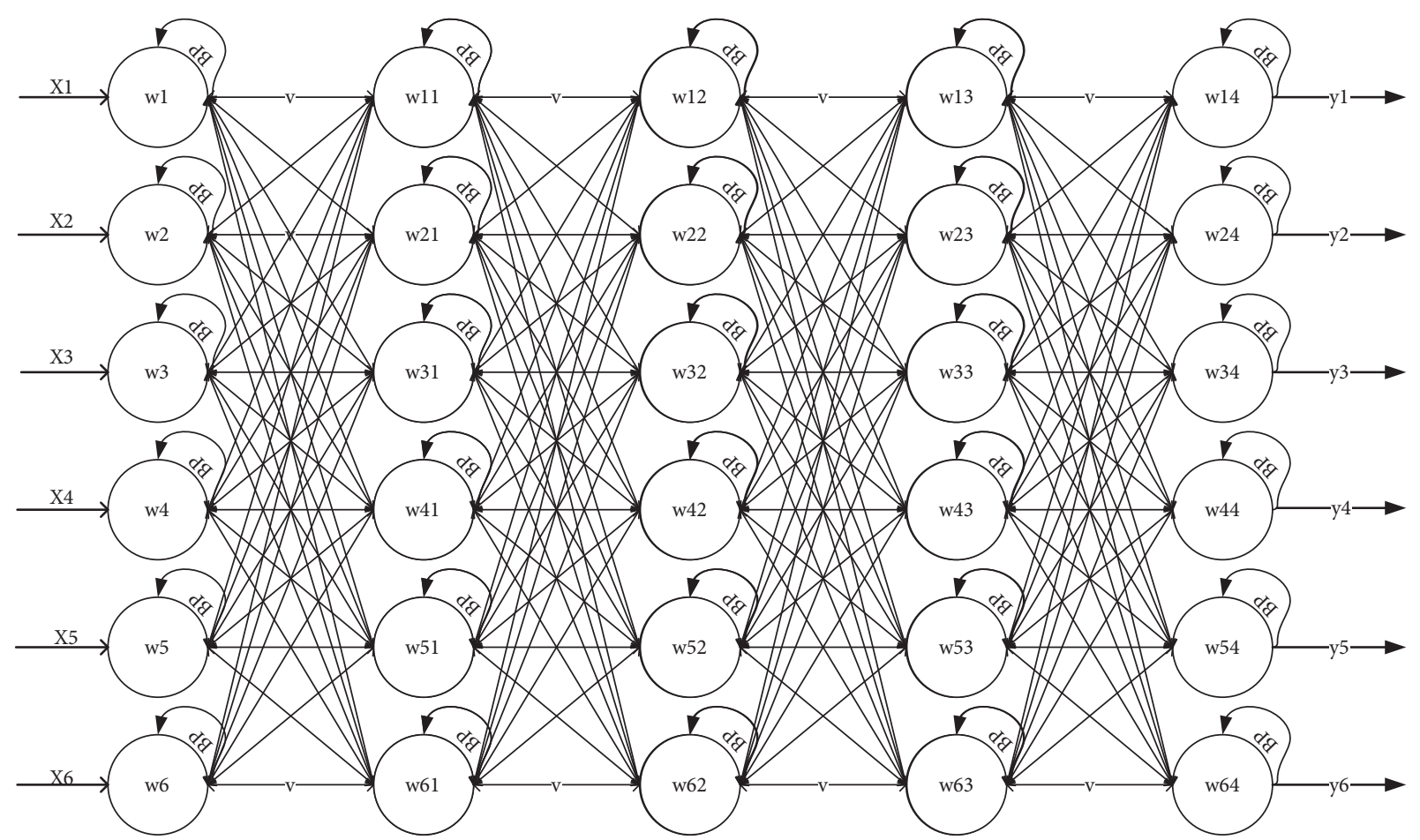

Figure 1: BP neural network structure.

denoted as Item3 $=[1,0,2,0,1,1,2]^{T}$. Suppose here we want to predict User's predicted value for Item 3; then, the input training data samples are the rating frequencies of Item 1 and Item 2, and the desired output value is the target User's true ratings of Item 1 and Items 2 and 3. After learning and training the prediction are done using the obtained BP neural network model, the input value is Item $3=[1,0,2,0,1,1,2]^{T}$ and the final output value of the $\mathrm{BP}$ neural network model is the predicted rating of User for Item 3. The pseudocode of the BP neural network training algorithm is shown in Table 1.

Similarly, we looked at the Resnick formula and discovered that it only takes into account the users' rating records, leaving out the implicit influence of similar users' ratings and the relationship between users. Without having to show and explain the mathematical equations of mapping relationships, a BP neural network can learn many inputoutput patterns mapping relationships and store them in the form of structural parameters, making it ideal for exploring user relationships. To address the abovementioned shortcomings of the Resnick formula, we propose using the BP neural network instead of the Resnick formula for target user rating prediction [17]. The use of a BP neural network has the advantage of being able to dig deeper into the details of user ratings, resulting in more accurate recommendations.

The construction of the BP neural network model requires determining how to obtain the input and output feature vectors. We first use the frequency of different ratings for each item in the set of similar users as the input feature vector for the optimal rating prediction module of the BP neural network, ignoring items that are not rated. The number of hidden layer nodes selected in the experiment are
$6,7,8$, and 9 , respectively. To ensure the accuracy of the experiment, the number of iterations and learning rate during data filling and neural network training are set to larger and smaller values, respectively.

$$
\operatorname{ROS}=\frac{N_{\text {words }}}{t-t_{s}},
$$

where $N_{\text {words }}$ denotes the total number of words included in the test taker's speaking, $t$ denotes the total duration of the speaking recording, and $t_{\mathrm{s}}$ denotes the duration of silence in the recording. In addition to the speech rate feature, the number of silences in the recording likewise reflects the test taker's speaking fluency to some extent. In terms of pronunciation quality evaluation, the pronunciation posterior probability feature is used by many speaking scoring systems and is also used in this paper to describe the accuracy of test takers' pronunciation.

Adherence to the principle of varied assessment methods in the teaching process aids in comprehensively judging students' learning effects and promoting their overall development. Diagnostic, formative, and summative assessment can be organically combined; not only should the teacher be involved in the evaluation process, but so should the students; and a variety of evaluation methods, including student mutual evaluation, teacher-student mutual evaluation, and student self-assessment, can be used. Teachers can perform a diagnostic evaluation of students' prestudy before class based on their platform login situation and prepare for the class; teachers can complete formative evaluation of students based on their platform login situation, course learning hours, question and answer situation, test situation, 
TABLE 1: Improvements to the data sparsity problem.

Input: Training sample, expected output value $R$, maximum number of training times: $G_{\max }$, number of iterations gen, learning rate learn Output: Predicted value FR

1. While gen $<G_{\max }$

2. If $g e n=1$

3. Initialize neural network parameters $w, b$

4. Else

5. Fix $w, b$

6. $w_{i}=w_{j}-$ learn $* R(1-R) x_{i} \sum_{i}^{n} w_{i} \varepsilon$

7. Gen $=$ gen +1

8. End

and so on; students can evaluate their peers based on their peers' performance in group collaborative learning, and so on; students can complete self-evaluation based on their platform login situation, course learning hours, question and answer situation, test situation; finally, teachers can make summative assessments based on homework completion, overall mastery of knowledge, and so on; students can assess teachers based on the number of question and answer sessions, the organization of teaching activities, and so on. Therefore, the use of diversified evaluation methods can enhance learners' participation in teaching activities, improve learners' learning motivation, and thus promote education and teaching reform, as shown in Figure 2.

Intelligent speaking scoring refers to the dynamic process from audio input to total score output. In the scoring system designed in this paper, the process can be described in Figure 2. The candidate's speaking recordings are first processed by the speech noise reduction module for noise reduction, followed by the speech recognition engine which transcribes the clean recordings into the corresponding textual content. Generally, features with a correlation coefficient lower than 0.2 should not be selected. The intelligent scoring system should describe the examinee's spoken language from multiple dimensions. Features with high similarity should not be included at the same time, so we calculate the cross-correlation between the selected features, for each pair of features with a correlation coefficient greater than 0.9 . Then, the data processing module extracts speechlike features and text-like features from the clean recordings and the speech recognition text and inputs the two types of features into the speech scoring model and the text scoring model, respectively. Finally, the outputs of the two scoring models are summed to obtain the total score. The general scoring system is designed to fit the feature values by one scoring model, and the design of this paper using two scoring models (speech scoring model and text scoring model) is mainly to improve the accuracy of the scoring system. Also, in a real-life marking environment, teachers assess candidates' speaking at the speech and content (text) levels separately. Therefore, such a design is consistent with the manual scoring approach.

\subsection{College English Prediction Skills Construction. When} faced with the task of learning English reading, most students will revert to rote learning and gradually lose the ability to comprehend the reading problems. Students, for example, pay more attention during the listening process to the grammar points, sentences, or passages that the teacher asks them to memorize, and students are busy taking notes that the teacher emphasizes when explaining words, testing vocabulary, or analyzing the structure of passages, and active class participation is misinterpreted by students as not missing notes and missing various language points. In addition, students understood the text in isolation, ignoring the connections between sentences [18]. This inhibits students' thinking skills to some extent, making critical thinking and applying what they've learned to real-life situations more difficult.

In general, there are four types of relationship mining: association rule mining, correlation mining, sequential pattern mining, and causal data mining. In association rule mining, the goal is to find the if-then rule of the form; namely, if a set of variable values is found, another variable will usually have a particular value. In sequential pattern mining, the goal is to find temporal associations between events and determine the path of student behavior leading to the final learning interest event. The goal of causal data mining is to find whether one event is the cause of another event, assuming if an instructional event is randomly selected using an automated experiment and often leading to a positive learning outcome.

There are four types of relationship mining: association rule mining, correlation mining, sequential pattern mining, and causal data mining. Simply put, it is a sentence that does not conform to the rules of grammar. It is usually found in the compositions of learners of English as a second language and is a common mistake made by beginners, mainly in tenses, plural forms, use of various subordinate clauses, etc. These errors are also commonly found in the compositions of learners of English as a second language. These errors are mainly in the form of verb misuse, which is usually correct in terms of grammatical rules, but from the semantic point of view, the improper use of verbs in their context causes semantic confusion, as shown in Figure 3.

From the identity of the user who uses it, one can see personalized recommendations as an efficient information filter that helps one make choices, it collects our historical behavioral data, ratings of products, click-through rates of products, and other information and organizes them, and then it uses mathematical methods such as data mining to perform data analysis on the collected information to predict which products we are most likely to like and provide us with more product choices, thus helping us in quick selection or decision making. 


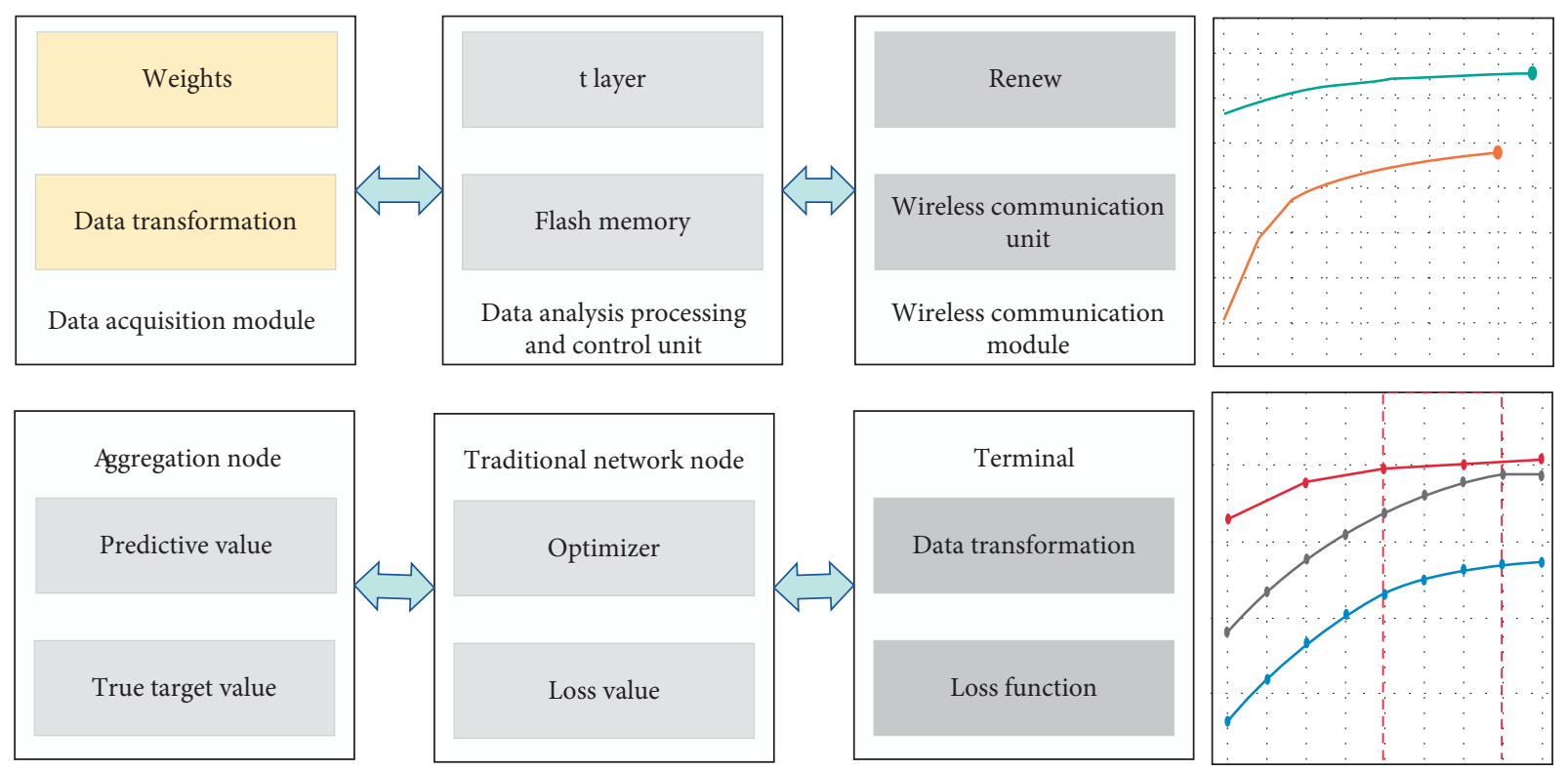

FIGURE 2: Algorithm working principle.
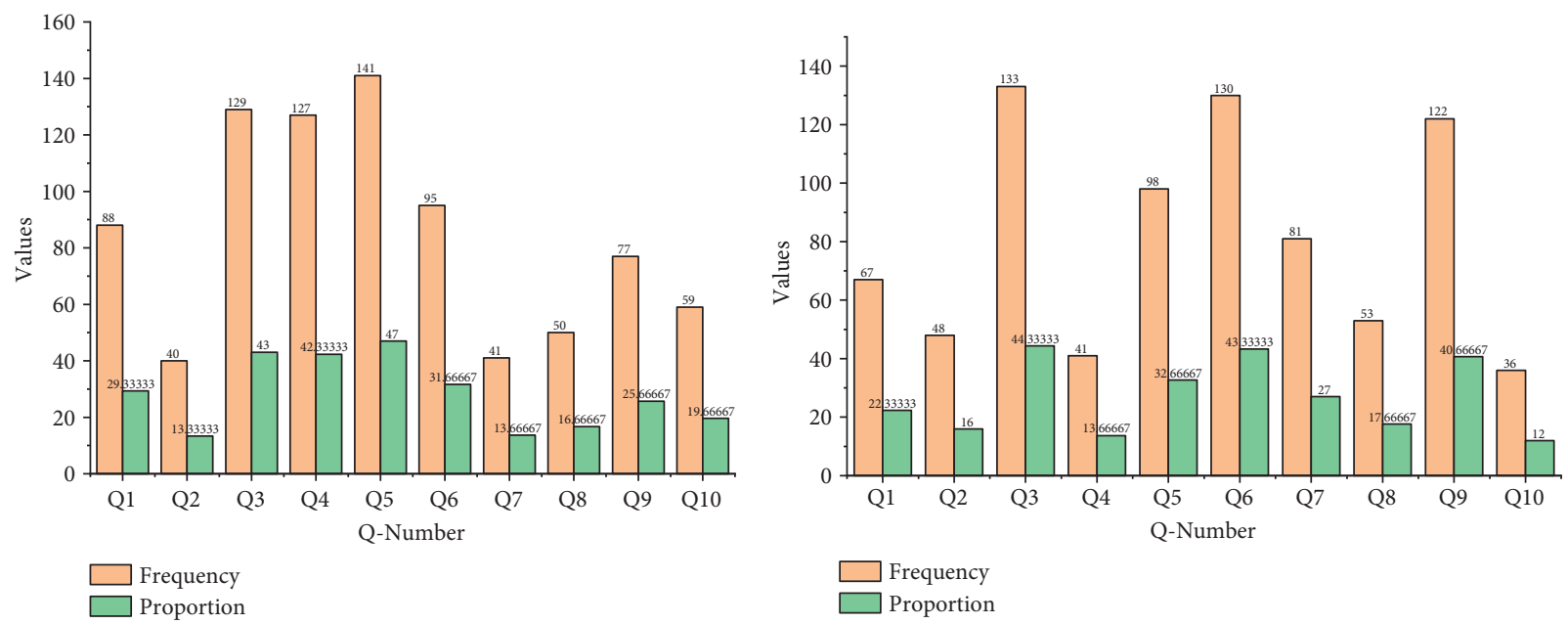

(a)

(b)
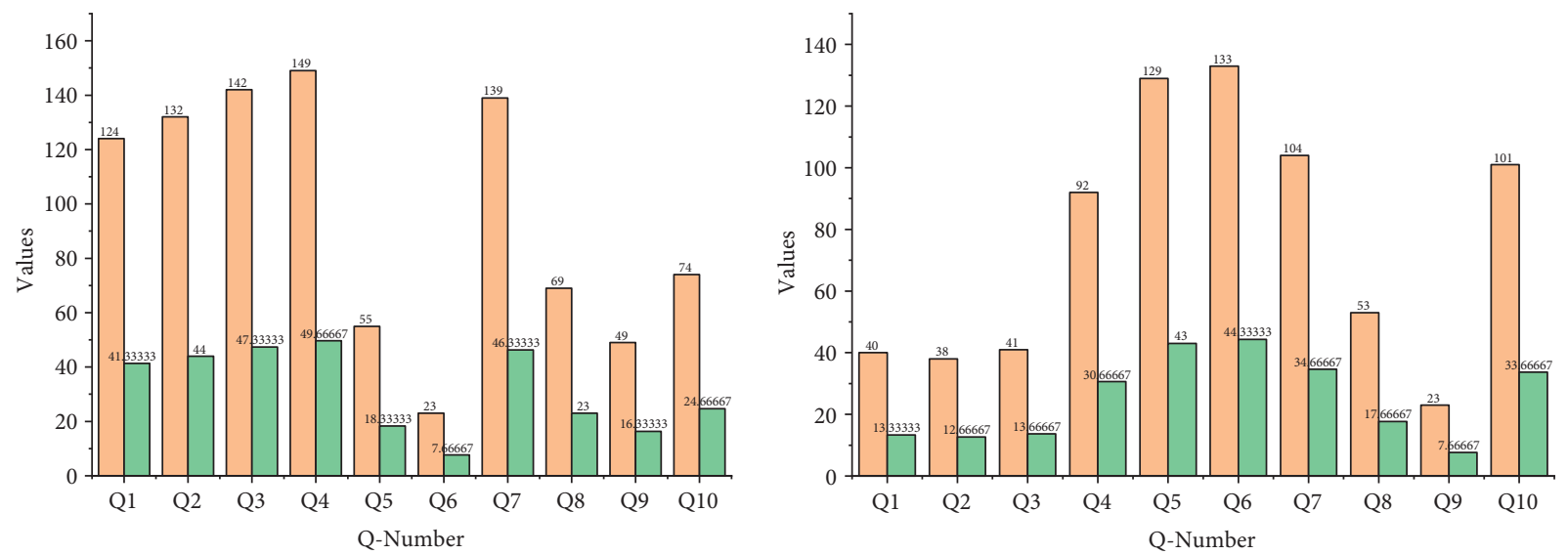

$\square$ Frequency

$\square$ Proportion

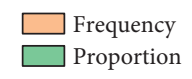

(c)

(d)

FIgURE 3: Effectiveness and the impact of beliefs. 


$$
s\left(u_{a}, u_{b}\right)=\frac{\sum_{i_{c} \in i_{a b}}\left(r_{a c}+r_{a}\right)\left(r_{b c}+r_{b}\right)}{\sqrt{\sum_{i_{c} \in i_{a b}}\left(r_{a c}-r_{a}\right)\left(r_{b c}-r_{b}\right)} \sqrt{\sum_{i_{c} \in i_{a b}}\left(r_{a c}+r_{a}\right)\left(r_{b c}+r_{b}\right)}},
$$

where $r_{a c}$ and $r_{a}$ are the average ratings of the rated items for user $u_{a}$ and user $u_{b}$, respectively. The inaccuracy caused by the scaling problem is reduced by subtracting the average of each user's rating of the item. From the analysis of the identity of the enterprise, the personalized recommendation is a powerful tool to understand the needs of our users, which can help us to provide the most demanded products according to the users' needs, thus winning users for the enterprise and improving the competitive advantage among other enterprises. Personalized recommendations are now used in many e-commerce platforms, and some platforms even introduce more than one algorithm, for example, "Dangdang" users will not only show links to information about other brands of mobile phones at the bottom of the purchase page but also show the number of people who bought the phone next to the information link [19]. Multiple evaluation methods such as student mutual evaluation, teacher-student mutual evaluation, and student selfevaluation can be carried out. Teachers can make diagnostic evaluations of students' preview conditions before class to prepare for class based on the login status of the student platform. This information gives the consumer a reference for purchasing, thus making it easier for the user to choose the product.

Suppose there are items $A, B, C$, and $D$ and three users $X$, $\mathrm{Y}$, and $\mathrm{Z}$. $\mathrm{X}$ likes items $\mathrm{A}, \mathrm{B}$, and $\mathrm{C}$; Y likes items A and B; Z likes item D. All these users rate their favorite items; if we want to recommend items to user $\mathrm{Y}$, then we first calculate the similarity between the target user and other users by the similarity formula and filter out the set of users with the most similar interests to the target user, similarity. The formula will be described in detail later. From the example we can see that user $\mathrm{X}$ and user $\mathrm{Y}$ have similar interests, so we can consider that user $\mathrm{X}$ is a similar neighbor of user $\mathrm{Y}$, and their interests are closer. Finally, the items that the neighboring users like but the target user has not participated in in the evaluation are recommended to the target user; user $\mathrm{x}$ likes item $\mathrm{C}$ but user $\mathrm{Y}$ does not have a rating for it, so item $\mathrm{C}$ is recommended to user $Y$. In practice, there are many items; generally we go through the Resnick rating formula to calculate the target user's rating of unrated items and then sort the ratings. The top $\mathrm{N}$ items with the highest ratings are recommended to the target user, as shown in Figure 4.

The user-based collaborative filtering recommendation algorithm is suitable for scenarios where items change quickly, such as news applications, based on the above principles. However, when dealing with a large number of users, calculating user similarity can be difficult, and user interests change over time, making hidden features such as interests difficult to capture and the process of change fast and difficult to record. In this case, a collaborative filtering algorithm based on items can be considered. The item-based collaborative filtering algorithm is best suited for scenarios in which the number of items is much lower than the number of users, and it performs better for recommendations in areas with a large variety of long-tail items.

\subsection{Experimental Design of English Reading Efficiency.} Feature extraction is a very important step before performing machine learning, which determines the confidence and accuracy of the scoring model. If the features are selected accurately enough, even if the neural network model behind is not optimal, a scoring result with less error can still be obtained. In the BP neural network-based scoring model studied in this paper, two main types of features are extracted: speech-like features (pronunciation, fluency, effective duration of spoken language) and text-like features (text length, topic relevance, grammar, lexical richness, sentence structure). Speech-like features are extracted directly through the speech signal, and text-like features are extracted through the output of the speech recognition engine.

The expected output value is the actual scores 2 and 3 of the target user for Item 1 and Item 2, and the BP neural network model obtained is used to predict after learning and training. A feature screening exercise is often also required before features can be extracted using correlation techniques. The importance of each feature can be measured by calculating its Pearson correlation coefficient with manual scoring, and usually, no feature should be selected with a correlation coefficient below 0.2. An intelligent scoring system should describe the candidate's speaking in multiple dimensions, and features with high similarity should not be included at the same time, so we calculate the intercorrelation between the selected features and remove one of them for each pair with a correlation coefficient greater than 0.9. Since this paper targets open-ended speaking scores, there is usually no fixed reference answer for such questions, so when doing feature selection in this paper, we mainly choose generic features rather than features that are strongly correlated with reference text answers, except for semantic similarity features, as shown in Figure 5.

Effectiveness evaluation strategies involve learners analyzing and judging their reading learning outcomes based on reading course learning objectives or self-established reading learning program goals and making self-regulation accordingly. Evaluation of reading learning outcomes refers to analyzing the characteristics of the reading material that has been studied and the individual's reading comprehension skills. Students are expected to consciously and frequently evaluate their reading results and test the achievement of goals concerning established objectives [20].

Comprehensive, timely, and effective learning support is a key factor in assisting learners to complete their online English reading learning. It can effectively reduce learners' anxiety levels in facing difficulties. Learning support consists 

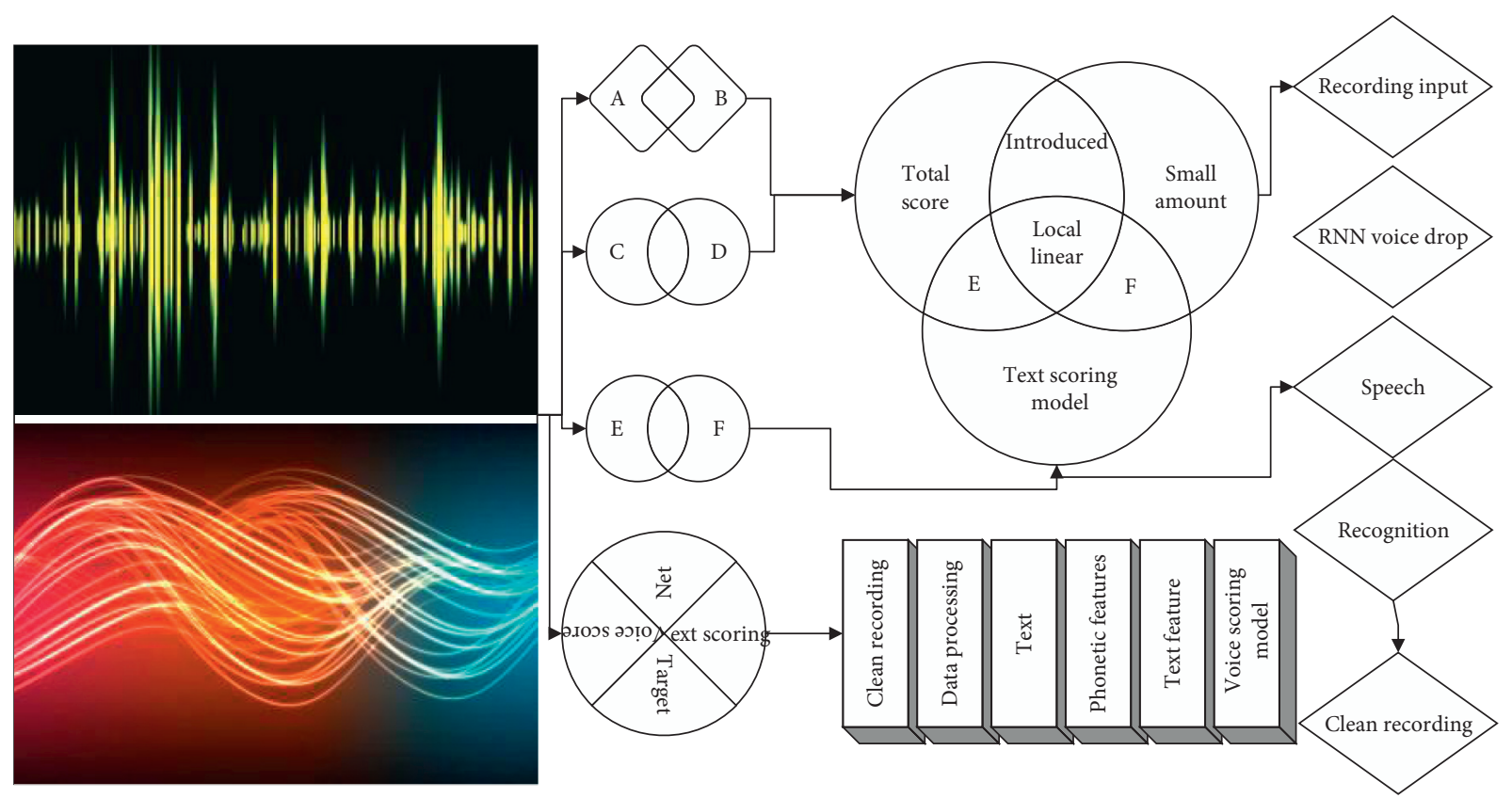

FIGURE 4: System scoring process.
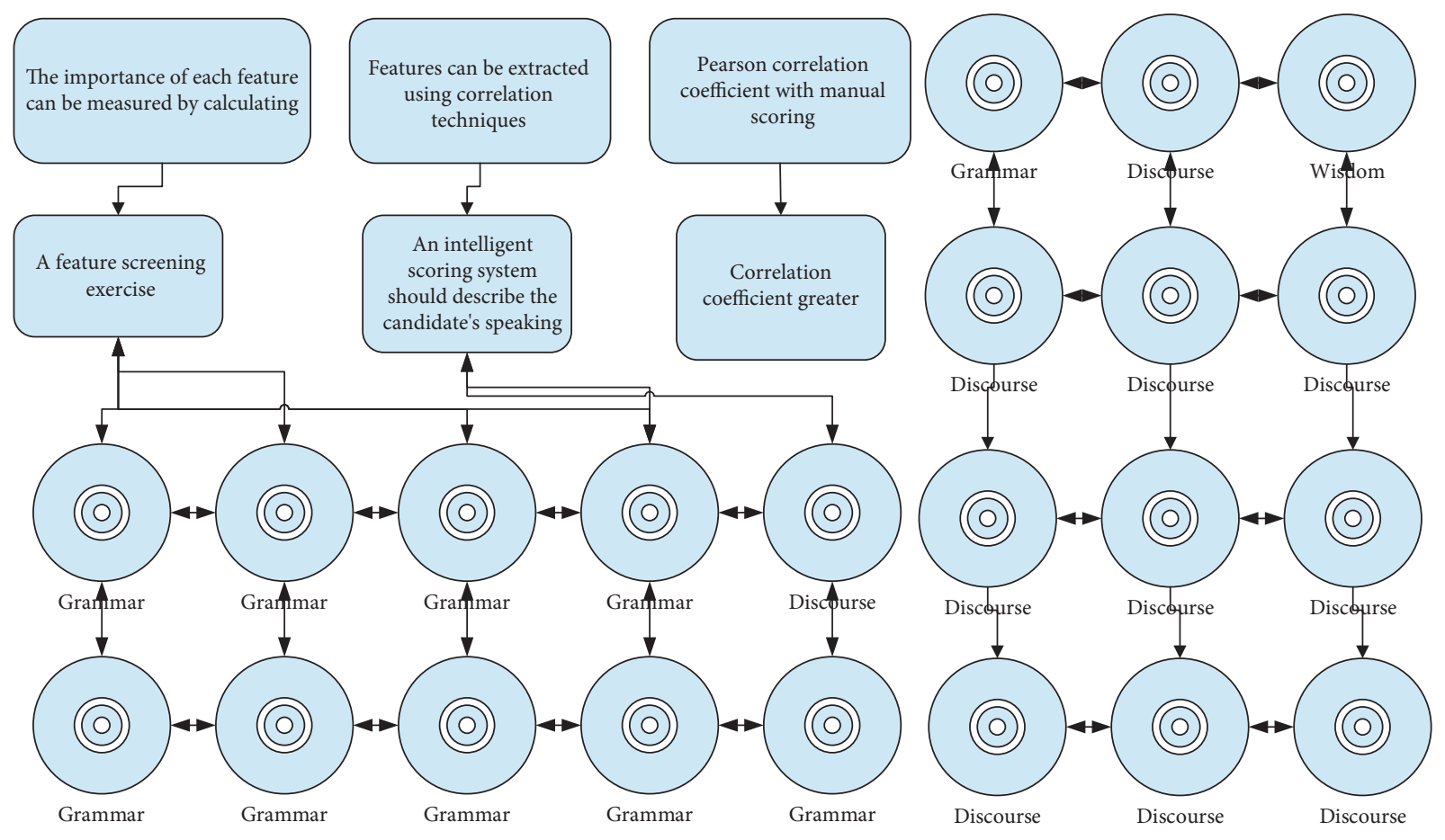

FIGURE 5: Experimental framework for reading efficiency.

of two components: support services and support tools. Support services refer to online academic tutorials, question and answer sessions, discussions, and evaluation of results and can be provided by computers, teachers, or fellow students. Finally, the output layer is processed to output the prediction results; the second stage: if the error between the predicted value and the actual value obtained through the output layer does not reach the preset accuracy, the error information will be fed back inversely, and the weight of the information will be transmitted. The bias is adjusted layer by layer. Support tools are computer network technologies and software, such as online dictionaries, audio and video players, and online timers. These services and tools constitute a comprehensive learning support system.

The multimodal reading instruction model is more effective than the traditional "blackboard-textbook" reading 
instruction model in improving students' reading comprehension. They argue that multimodal teaching stimulates students' multiple senses, greatly enriches and enhances the comprehensible input of information, and is more in-depth than single-modal language explanations, which can improve students' memory and ability to comprehend and construct the meaning of the discourse. As a result, the researcher came to the conclusion that combining multimodal instruction with traditional reading instructional methods is a good way to improve students' reading achievement and comprehension skills.

\section{Analysis of Results}

4.1. Optimized BP Network Performance Results. We need to determine the number of neural nodes in the input, implicit, and output layers of the BP neural network used. As mentioned earlier in Section 3, the input vector of the BP neural network uses the frequency of different ratings for each item and contains the number of users with ratings from 1 to 5 and the number of users with no ratings, a total of six feature values, so we use six nodes for the input layer. The output of the BP neural network is the prediction of a singleitem prediction value, so one number of nodes is used for the output layer. The choice of the number of nodes in the hidden layer has an important impact on the performance of the neural network; too few nodes may result in not having enough connection weight combinations to learn several samples, and too many nodes may reduce the generalization ability of the network after training. However, no one has yet discovered a sound theoretical method to guide the selection of BP neural network structures, which can generally only be selected empirically. Therefore, we use experimental observations to select the number of nodes in the implicit layer with relatively good results as the basis for subsequent experiments.

The number of nodes in the hidden layer was chosen to be $6,7,8$, and 9 to ensure the accuracy of the experiments, the number of iterations and learning rate were set to larger and smaller values for data filling and neural network training, respectively, and the test results of the three improved recommendation algorithms are shown in Figure 6.

It is often used for nonlinear, classification, and other problems in which it is difficult to obtain internal laws through observation. Therefore, neural networks are often used in the field of recommendation. The experiments show that the improved Pearson recommendation algorithm is slightly less effective than the other two recommendation algorithms, where the BP-P-asks algorithm has the best results. The accuracy of the recommendation results of the three improved recommendation algorithms showed a trend from low to high to low, where all of them had the smallest MAE value when the number of nodes in the hidden layer was 7 , namely, the highest accuracy was achieved, so this optimal empirical value was used as the number of nodes in the hidden layer for constructing the BP neural network in the subsequent experiments.

The number of iterations and the learning rate have an impact on the results when using gradient descent to optimize populated data; too few iterations will result in insufficient accuracy, and too many will take a long time, which is intolerable in practice, so a suitable value must be set. Similarly, a learning rate that is too low will take too long to converge, while a learning rate that is too high will result in oscillation around the minimum but not convergence to the minimum. As a result, the optimal number of iterations and learning rate are determined through experimentation. The BP neural network's training number is set to 1000 , the learning rate is set to 0.008 , the minimum error is set to 0.1 , and the regular term parameter is set to 0.025 to ensure the best results. The target user's nearest-neighbor users are limited to 50 . In the growth of $50, \ldots, 300$, the number of iterations for data filling is set to 50,100 , and 150 , and the learning rate is set to $0.006,0.007$, and 0.008 . Figure 7 shows the results of the experiments on BP-P-pfc, BP-P-csc, and $\mathrm{BP}-\mathrm{P}-\mathrm{acsc}$, respectively. It is impossible to truly master and apply what has been learned. Online courses compensate for the shortcomings of classroom teaching by providing students with more learning options and targeted training on reading strategies, allowing them to fully utilize their reading initiative and creativity.

The BP-P-pfc, BP-P-csc, and BP-P-acsc algorithms perform best when the number of neighbors is 40,40 , and 30 , respectively, and the BP-P-acsc algorithm is more accurate than the other two improved algorithms, which is due to the similarity calculation formula of the BP-P-acsc algorithm taking into account the problem of scoring range based on BP-P-csc and making improvements. We also discovered that, at any number of neighbors, the accuracy of the three improved recommendation algorithms is much higher than that of the unimproved algorithm. While the improved Pearson recommendation algorithm is slightly less accurate than the other two improved recommendation algorithms, the recommendation quality is still higher than that of the original Pearson recommendation algorithm.

4.2. Analysis of Experimental Results. When designing prereading activities, they should consider whether they can help students activate or build up relevant background knowledge, help them remove some language barriers, or help them predict the content of the text while stimulating their interest, to design activities that not only are compelling and interesting but also value the relevance to the content of the text and the teaching objectives. When helping students activate relevant background knowledge or predict the content of the text, students' life experiences and existing background knowledge should be considered. Teachers should not take for granted that students should know so-and-so, which would easily lead to silence in the prelesson activities. At this time, the students' reading focus will gradually shift from focusing on learning to using English to obtain information and experience the true purpose of English reading. In the activation session of cultural background knowledge, considering that most students lack personal experience in this area and the explanation is rather abstract, teachers can use pictures, objects, videos, and other visual ways and present them to 


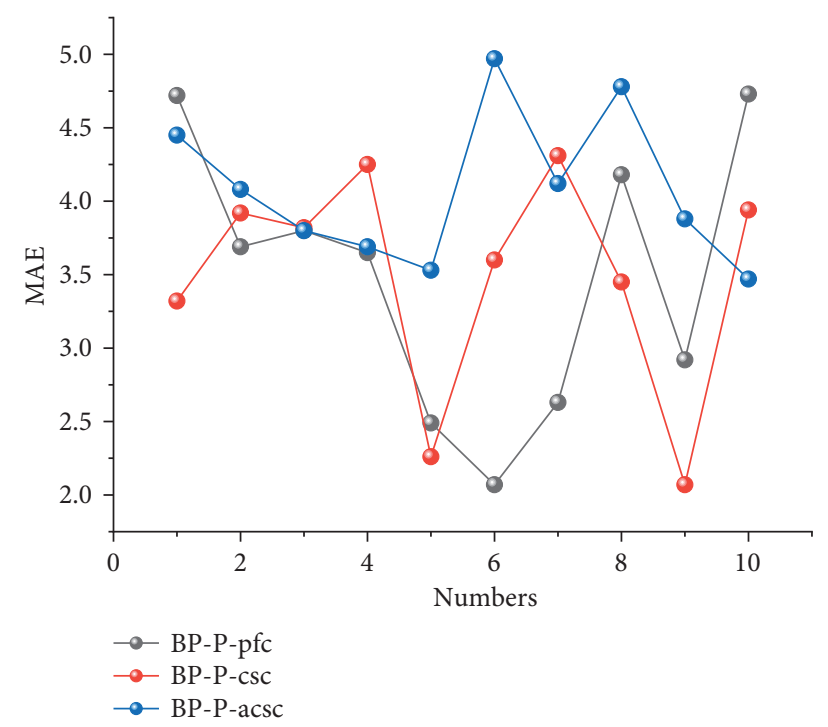

FIgURE 6: Effect of the number of nodes in the hidden layer on the results.

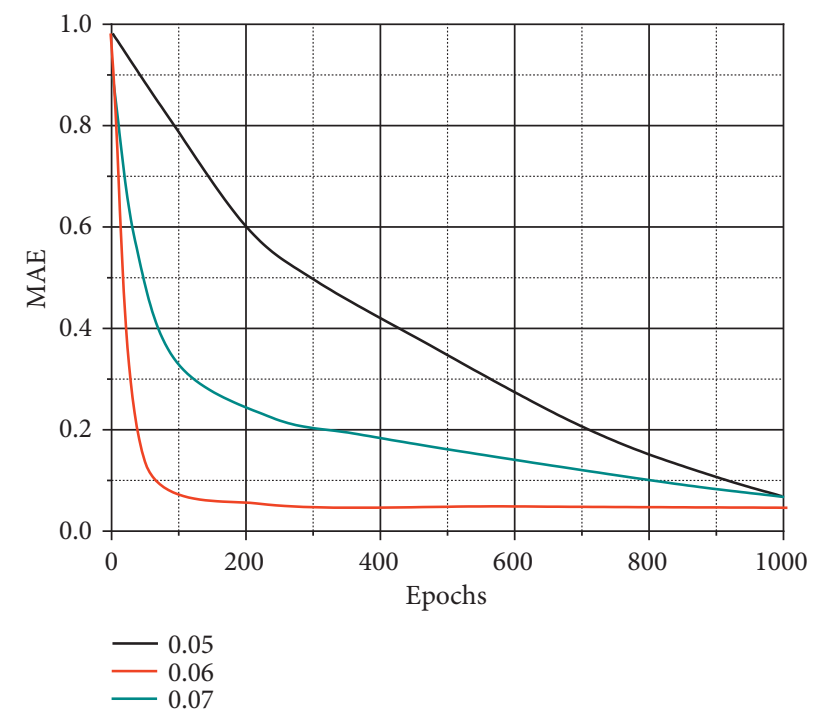

FIGURE 7: Effect of number of iterations and learning rate on BP algorithm.

students vividly and activate a series of low-level schemas for reading. If a game session is used, which is an easy way to increase students' motivation, there will be more students who want to participate. In addition to paying strict attention to the game's topic and the textbook's theme, it is also important to keep the speed under control, as seen in Figure 8.

Students would passively listen to lectures, answer questions raised by the teacher, and even more passively be asked by name to stand up and answer questions raised by the teacher in the traditional teaching model, which is typical of students' passive learning. Students' ability to actively question in class is a significant step forward in improving their ability to learn independently, to aid in the development of students' comprehensive language ability, cultivate cultural awareness, develop English reading habits, and master certain English reading skills. The percentage of this indicator in the experimental class reached 94.1 percent after data analysis, indicating that the students' ability to learn independently has improved significantly. Students can actively think in the classroom, follow the teacher's guiding ideas, fully integrate the teacher's teaching and learning, and dare to question. Teachers must properly guide and protect students' inquisitive nature in order to foster a more relaxed environment that encourages them to ask more questions. Although 55.7 percent of the students in the control class also questioned in class, the percentage was still lower than that of the experimental class, and 44.3 percent of the control class students either did not know how to question in class or did not approve of questioning at all, demonstrating that the experimental class students were more capable of independent learning in class than the control class. 


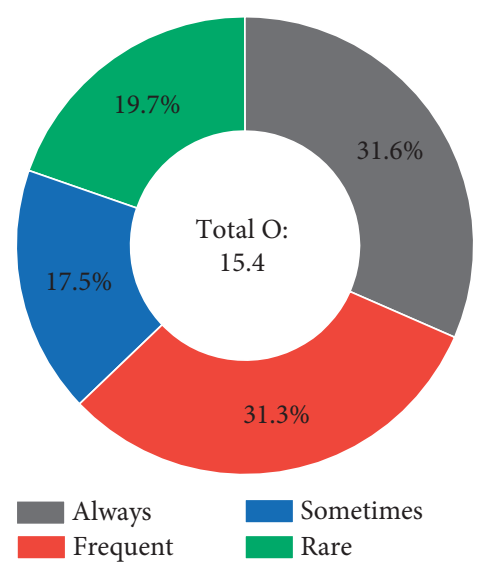

(a)

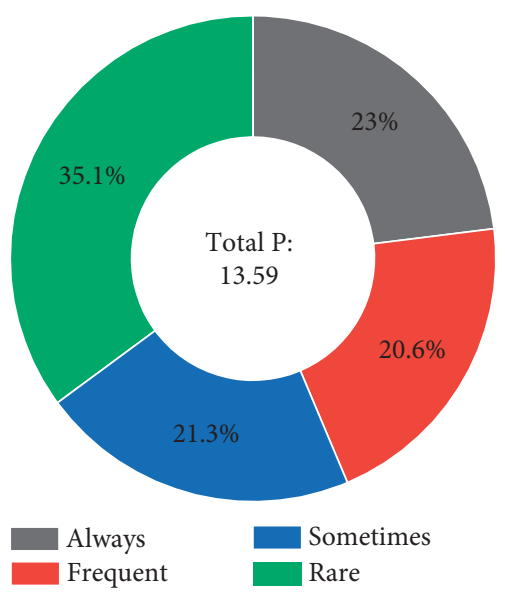

(b)

Figure 8: Experimental comparison results.

As the ordinary essays were selected from the Kaggle standard dataset, the dataset deleted part of the content to protect privacy, which in turn led to reading barriers, the total number of invalid evaluation essays were deleted, the remaining 35 essays were input into the system and output machine comments, the machine comments were divided into 5 grades, the teachers were evaluated in the evaluation of words, grammar, and logical structure, and sentence coherence was also divided into 5 grades, after collecting the results. After collecting the results and inputting them for analysis, independent sample $t$-test and consistency kappa test was chosen to test the consistency between machine comments and manual comments. The independent sample $t$-test is shown in Figure 9.

There was no significant correlation between the teacher rubric and the machine rubric in terms of vocabulary, grammar, sentence coherence, and logical structure of all aspects of the rubric. The consistency test result kappa $=0.803$ indicated that the machine rubric had high consistency with the human rubric. When the user has a relatively clear search goal, the search engine can provide great help, but when the user's goal is relatively vague, the search engine cannot better bring the user the desired result. The findings demonstrate that machine rubrics based on Coho-Metrix and fuzzy logic can generate descriptive evaluation feedback accurately and comprehensively and that machine evaluation feedback will replace manual feedback, free teachers from mechanical grading feedback, and improve the efficiency of teachers' instruction. A comprehensive and accurate visualization report was generated by combining theories of writing feedback and data visualization, and the accuracy of the machine rubric was experimentally verified to improve the interactive feel of the automatic composition scoring system.

\section{Conclusion}

In this paper, we design and implement an open speaking scoring system that can help teachers reduce their workload by automating the marking of candidates' speaking

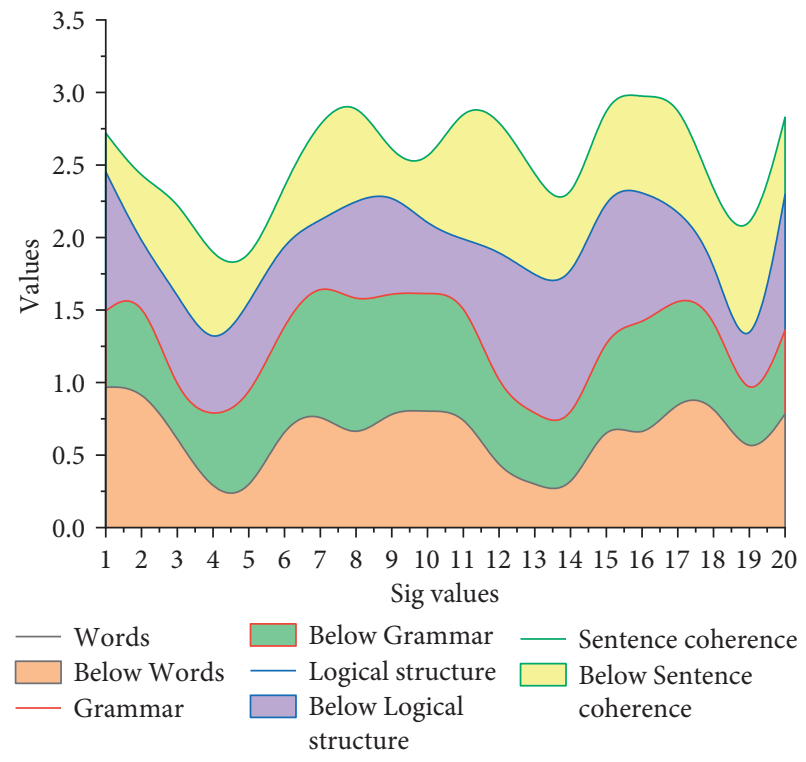

FIGURE 9: Independent samples $t$-test.

recordings. The experimental results show that the $\mathrm{BP}$ network model has some practicality and achieves better scoring performance with a smaller training dataset. This project designs and develops a college English reading online course based on the process and standard requirements of online course instructional design, with the goal of increasing learners' awareness and skills in English reading strategies. The focus of the design process is on incorporating macro theories into practical teaching, integrating reading strategy training with reading course teaching, and attempting to develop a process for designing and implementing English reading online courses to better assist learners' self-learning and classroom teaching. This necessitates the researcher's continued promotion of the teaching model's practical application in future educational practice, not only in his own classroom but also in experimental schools as capacity allows, in order to obtain more 
experimental teaching data, improve the teaching model, and improve the credibility and effectiveness of its promotion. Nonetheless, in the actual research process, the final research results must be summarized and adjusted as needed, and the designed online courses must be subjected to more systematic application studies to verify their true feasibility and effectiveness.

\section{Data Availability}

The data used to support the findings of this study are available from the author upon request.

\section{Conflicts of Interest}

The author declares no conflicts of interest.

\section{Acknowledgments}

The author acknowledges the project of Heilongjiang Province's Education Science, "Research on Metaphor Translation Strategy and Teaching Application in Political and Economic Texts in Belt and Road" (project no. GJC1318022) and Funds for Basic Scientific Research in University, "Research on Metaphor Translation Strategies in "Analysis of China" from the Perspective of One Belt and One Road" (project no. 3072019CFW1205).

\section{References}

[1] Y. K. Semero, D. Zheng, and J. Zhang, "A PSO-ANFIS based hybrid approach for short term PV power prediction in microgrids," Electric Power Components and Systems, vol. 46, no. 1, pp. 95-103, 2018.

[2] Z. Tian, Y. Ren, and G. Wang, "Short-term wind speed prediction based on improved PSO algorithm optimized EMELM," Energy Sources, Part A: Recovery, Utilization, and Environmental Effects, vol. 41, no. 1, pp. 26-46, 2019.

[3] Q. Chen, Y. Song, and J. Zhao, "Short-term traffic flow prediction based on improved wavelet neural network," Neural Computing \& Applications, vol. 33, no. 14, pp. 81818190, 2021.

[4] K. Shi, Y. Qiao, W. Zhao, Q. Wang, M. Liu, and Z. Lu, “An improved random forest model of short-term wind-power forecasting to enhance accuracy, efficiency, and robustness," Wind Energy, vol. 21, no. 12, pp. 1383-1394, 2018.

[5] M. Ali Ghorbani, R. Kazempour, K.-W. Chau, S. Shamshirband, and P. Taherei Ghazvinei, "Forecasting pan evaporation with an integrated artificial neural network quantum-behaved particle swarm optimization model: a case study in Talesh, Northern Iran," Engineering Applications of Computational Fluid Mechanics, vol. 12, no. 1, pp. 724-737, 2018.

[6] D. Zheng, Y. K. Semero, J. Zhang, and D. Wei, "Short-term wind power prediction in microgrids using a hybrid approach integrating genetic algorithm, particle swarm optimization, and adaptive neuro-fuzzy inference systems," IEEJ Transactions on Electrical and Electronic Engineering, vol. 13, no. 11, pp. 1561-1567, 2018.

[7] K. Gu, Y. Zhou, H. Sun, L. Zhao, and S. Liu, "Prediction of air quality in Shenzhen based on neural network algorithm,"
Neural Computing \& Applications, vol. 32, no. 7, pp. 18791892, 2020.

[8] M. Baek, F. DiMaio, I. Anishchenko et al., "Accurate prediction of protein structures and interactions using a threetrack neural network," Science, vol. 373, no. 6557, pp. 871-876, 2021.

[9] F. S. Hoseinian, B. Rezai, E. Kowsari, and M. Safari, "A hybrid neural network/genetic algorithm to predict $\mathrm{Zn}$ (II) removal by ion flotation," Separation Science and Technology, vol. 55, no. 6, pp. 1197-1206, 2020.

[10] F. Jabeen, M. Maqsood, M. A. Ghazanfar et al., "An IoT based efficient hybrid recommender system for cardiovascular disease," Peer-to-Peer Networking and Applications, vol. 12, no. 5, pp. 1263-1276, 2019.

[11] P. Taherei Ghazvinei, H. Hassanpour Darvishi, A. Mosavi et al., "Sugarcane growth prediction based on meteorological parameters using extreme learning machine and artificial neural network," Engineering Applications of Computational Fluid Mechanics, vol. 12, no. 1, pp. 738-749, 2018.

[12] Q. Liu, Y. Zou, X. Liu, and N. Linge, "A survey on rainfall forecasting using artificial neural network," International Journal of Embedded Systems, vol. 11, no. 2, pp. 240-249, 2019.

[13] S. Wei, Z. Chongchong, and S. Cuiping, "Carbon pricing prediction based on wavelet transform and K-ELM optimized by bat optimization algorithm in China ETS: the case of Shanghai and Hubei carbon markets," Carbon Management, vol. 9, no. 6, pp. 605-617, 2018.

[14] B. Cao, J. Zhao, Z. Lv, Y. Gu, P. Yang, and S. K. Halgamuge, "Multiobjective evolution of fuzzy rough neural network via distributed parallelism for stock prediction," IEEE Transactions on Fuzzy Systems, vol. 28, no. 5, pp. 939-952, 2020.

[15] S. Shankar, T. Mohanraj, and R. Rajasekar, "Prediction of cutting tool wear during milling process using artificial intelligence techniques," International Journal of Computer Integrated Manufacturing, vol. 32, no. 2, pp. 174-182, 2019.

[16] Y. Raptodimos and I. Lazakis, "Application of NARX neural network for predicting marine engine performance parameters," Ships and Offshore Structures, vol. 15, no. 4, pp. 443-452, 2020.

[17] Z. M. Yaseen, M. I. Ghareb, I. Ebtehaj et al., "Rainfall pattern forecasting using novel hybrid intelligent model based ANFIS-FFA," Water Resources Management, vol. 32, no. 1, pp. 105-122, 2018.

[18] Y. Li and W. Chen, "Entropy method of constructing a combined model for improving loan default prediction: a case study in China," Journal of the Operational Research Society, vol. 72, no. 5, pp. 1099-1109, 2021.

[19] W. Fang, Y. Guo, W. Liao, K. Ramani, and S. Huang, "Big data driven jobs remaining time prediction in discrete manufacturing system: a deep learning-based approach," International Journal of Production Research, vol. 58, no. 9, pp. 2751-2766, 2020.

[20] J. Yin, N. Wang, and A. N. Perakis, “A real-time sequential ship roll prediction scheme based on adaptive sliding data window," IEEE Transactions on Systems, Man, and Cybernetics: Systems, vol. 48, no. 12, pp. 2115-2125, 2017. 